\title{
Composición de nidos de aves silvestres en parques urbanos y periurbanos del centro del Estado
} de Veracruz, México

Composition of wild bird nests in urban and peri-urban parks of center of the state of Veracruz, Mexico

Flores-Peredo Rafael ${ }^{1}{ }^{凶}$, Zárate-Tirado Xóchitl ${ }^{1}$, Bolívar-Cimé Beatriz del Socorro ${ }^{1}$, Mella-Méndez Isac ${ }^{1}$, Rosas-Ronzón María del Coral ${ }^{1}$, Domínguez-Espinosa Paola Ivette ${ }^{1}$

${ }^{1}$ Instituto de Investigaciones Forestales, Laboratorio de Ecología, Universidad Veracruzana, CP 91070.

${ }^{\bowtie}$ Autor para correspondencia: peredofr@gmail.com

Recibido: $13 / 07 / 2018$
Aceptado: 12/09/2018

\section{RESUMEN}

La urbanización modifica la abundancia y tipo de materiales naturales usados por aves en la nidación. La incorporación de materiales antrópicos (de uso humano) al nido es riesgosa para los polluelos y el éxito reproductivo de las poblaciones. En este trabajo se evalúa la composición de nidos de aves silvestres en parques urbanos y periurbanos de Xalapa y Coatepec, Veracruz, México. Durante marzojulio 2017, se eligieron dos parques urbanos y tres periurbanos en Xalapa y uno urbano en Coatepec, donde se colectaron en general diez nidos inactivos por mes en cada parque. Los nidos fueron secados y los materiales separados y pesados en una balanza analítica considerando cuatro criterios: material vegetal (MVE), material de origen animal (MOA), material de sustrato (MSU) y antrópico (MAN). Por cada tipo de material se obtuvo el porcentaje relativo. Un ANOVA con interacciones de dos vías (tipo de material y tipo de parque) fue utilizado para establecer diferencias en los porcentajes relativos transformados a raíz cuadrada del arcoseno. Un ACP mostró grupos de los materiales usados. Sólo la composición de nidos fue diferente entre tipos de materiales $\left(\mathrm{F}_{3,1312}=2637.26, P<0.001\right)$ siendo el MVE y MSU mayormente representados $1.28 \pm 0.016$ y $0.24 \pm 0.016$ (media del arcoseno \pm EE). Dos componentes explicaron el 93.2\% de la variación. El CP1 (2.2203 eigenvalor) se relacionó con el peso del MOA y el MAN, y el CP2 (1.5077 eigenvalor) con el MVE. Se concluye que las aves usaron mayor MVE en sus nidos con fines estructurales y de revestimiento, independientemente del ambiente urbano o periurbano.

Palabras clave: antropización, aves, nidación, elección de materiales, urbanización.

\section{ABSTRACT}

Urbanization modifies the type and abundance of natural materials used bybirds in nesting. The anthropic materials incorporation (for human use) in the bird nest is risky for the chicks and the reproductive population's success. In this study was assessed the composition of wild bird nests between urban and periurban parks of Xalapa and Coatepec, Veracruz, Mexico. During March-July 2017, two urban and three peri-urban parks were selected in Xalapa and one urban park in Coatepec, ten inactive nests were collected per month in each park. These nests were dried and the materials separated and weighted in an analytical scale considering four criteria: plant material (MVE), material of animal origin (MOA), substrate material (MSU) and anthropic (MAN). For each type of material, 
the relative percentage was obtained. A two-way ANOVA (the type of material and type of park) was used to establish differences with the relative percentages transformed to square root of arcsine. A PCA analysis showed groups of the materials used. Only the nest composition was different between material types $\left(\mathrm{F}_{3,1312}=2637.26, P<0.001\right)$ being the MVE and MSU mostly represented $1.28 \pm$ 0.016 and $0.24 \pm 0.016$ (mean arcsine $\pm \mathrm{SE}$ ). Two components explained $93.2 \%$ of the variation. The PC1 (2.2203 eigenvalue) was related to the MOA and MAN weight, and the PC2 (1.5077 eigenvalue) with the MVE. Birds used more MVE in their nestsfor structural and lining purposes, regardless of the urban or peri-urban environment.

Keywords: anthropization, birds, choice of materials, nesting, urbanization.

\section{INTRODUCCIÓN}

Las aves constituyen un grupo biológico bioindicador del efecto de la urbanización (Niemella, 2000), que se refleja cuando éstas se ven afectadas por la disponibilidad de alimento y refugio, presencia de depredadores exóticos,y en el uso de materiales adecuados para la construcción de sus nidos (Estades, 1995). Este último proceso, constituye un elemento clave para evaluar el uso de hábitat y los requerimientos ecológicos de este grupo de vertebrados en uno de los momentos más importantes del reclutamiento de sus poblaciones, la reproducción (Collias y Collias, 1984). Cada tipo de material usado en la elaboración del nidotiene una función particular, variando en función al área donde habite el ave. En ambientes naturales, se ha documentado el uso de ramas, tallos, hojas, herbáceas, pelos, musgos y epífitas en la construcción de nidos (Quilodrán et al., 2012; Moreno et al., 2005) y en ambientes urbanos materiales antrópicos como cuerdas, cinta de plástico, elástico, vendas de gasa y alambre (Townsend et al., 2014). Estos últimos, pueden tener efectos negativos sobre los polluelos que pueden morir si los consumen (Parker y Blomme, 2007). El objetivo de este trabajo fue evaluar la composición de nidos de aves silvestres entre parques urbanos y periurbanos de Xalapa y Coatepec, Veracruz, México, con el fin de conocer si en general las avesusan más materiales antrópicos en áreas urbanas que en periurbanas lo cual puede afectar su éxito reproductivo. Se espera que los nidos de parques periurbanos estén conformados por mayor cantidad de materiales naturales que los urbanos.

\section{MATERIALES Y MÉTODOS}

Tres parques ecológicos urbanos (Macuiltépetl y Natura en Xalapa, Cerro de las Culebras en Coatepec) y tres periurbanos (El Haya, Francisco Javier Clavijero y Campus CADXalapa) fueron muestreados durante marzojulio del 2017 (Tabla 1). En cada parque fueron colectados 50 nidos inactivos $(n=300) \sin$ considerar la especie de ave silvestre, debido a la dificultad de contar con un número homogéneo de nidos por especie en función a la disponibilidad. Los nidos fueron secados (Bondia-López, 2014) y los materiales fueron separados y pesados de acuerdo su origen. Debido al gran número de materiales registrados (27 en total) se agruparon en cuatro categorías dependiendo su naturaleza (SuárezRodriguez et al., 2013): material vegetal (hongos, líquenes, ramas, hojas, musgo, flores, semillas, fibra, tallos, raíces, corteza vegetal), de origen animal (excretas, exoesqueletos de 
insectos, pelo, plumas, seda, huesos, piel de serpiente), de sustrato (arcilla y rocas) y antrópico (alambre, hilo, papel, plástico, tela, colillas de cigarro). El porcentaje relativo se obtuvo de los materiales conbase al peso total en gramos del nido en seco. Un análisis de varianza (ANOVA) con interacciones de dos vías (tipo de parque y tipo de material) fue usado para determinar diferencias entre los tipos de materiales usados en la construcción de nidos entre parques urbanos y periurbanos. Los valores de porcentaje relativo (variable respuesta) fueron transformados a la raíz cuadrada del arcoseno para asumir una distribución normal. Las comparaciones entre medias se realizaron mediante el procedimiento post hoc de Tukey. Un análisis de componentes principales (ACP) fue realizado también para identificar de manera descriptiva alguna agrupación entre los tipos de materiales usados (Zar,

1999).

Tabla 1. Ubicación geográfica de los parques analizados.

\begin{tabular}{ccccc}
\hline Parque & Ubicación & Área (ha) & $\begin{array}{c}\text { Altitud } \\
(\mathbf{m} . \mathbf{s . n} . \mathbf{m} .)\end{array}$ & Categoría \\
\hline Macuiltépetl & $19^{\circ} 32^{\prime} 54^{\prime \prime} \mathrm{N}, 96^{\circ} 55^{\prime} 15^{\prime \prime} \mathrm{O}$ & 32 & 1580 & Urbano \\
Natura & $19^{\circ} 30^{\prime} 52^{\prime \prime} \mathrm{N}, 96^{\circ} 53^{\prime} 21^{\prime \prime} \mathrm{O}$ & 104 & 1274 & Urbano \\
Cerro de las Culebras & $19^{\circ} 27^{\prime} 36^{\prime \prime} \mathrm{N}, 96^{\circ} 57^{\prime} 39^{\prime \prime} \mathrm{O}$ & 40 & 1279 & Urbano \\
El Haya & $19^{\circ} 51^{\prime} 71^{\prime \prime} \mathrm{N}, 96^{\circ} 94^{\prime} 24^{\prime \prime} \mathrm{O}$ & 16 & 1420 & Periurbano \\
Francisco Javier Clavijero & $19^{\circ} 29^{\prime} 00^{\prime} \mathrm{N}, 97^{\circ} 9^{\prime} 00^{\prime \prime} \mathrm{O}$ & 22 & 1280 & Periurbano \\
Campus CAD-Xalapa & $19^{\circ} 30^{\prime} 41^{\prime \prime} \mathrm{N}, 96^{\circ} 54^{\prime} 58^{\prime \prime} \mathrm{O}$ & 33 & 1352 & Periurbano \\
\hline
\end{tabular}

\section{RESULTADOS Y DISCUSIÓN}

Los datos no mostraron una interacción entre tipo de parque y tipo de materiales en la composición de nidos $\left(\mathrm{F}_{3,1312}=0.620, P=\right.$ $0.602)$ lo cual rechaza la hipótesis planteada. Sin embargo, la composición de nidos varió entre tipos de materiales $\left(F_{3,1312}=2637.26, P=\right.$ $<0.001$ ), siendo el material vegetal que se presentó de forma mayor en los nidos (1.28 \pm $0.016)$, así como la de sustrato $(0.24 \pm 0.016)$, pero no así el de origen animal y antrópico $(0.044 \pm 0.016$ y $0.048 \pm 0.016$, respectivamente; Figura 1). En el ACP se formaron dos componentes principales que explicaron el 93.2\% de la variación en función al tipo de material usado. El componente PC1 se relacionó con el peso del material de origen animal (MOA) y el antrópico (MAN), mientras que el PC2 tuvo una relación alta con el peso del material vegetal (Tabla2). 


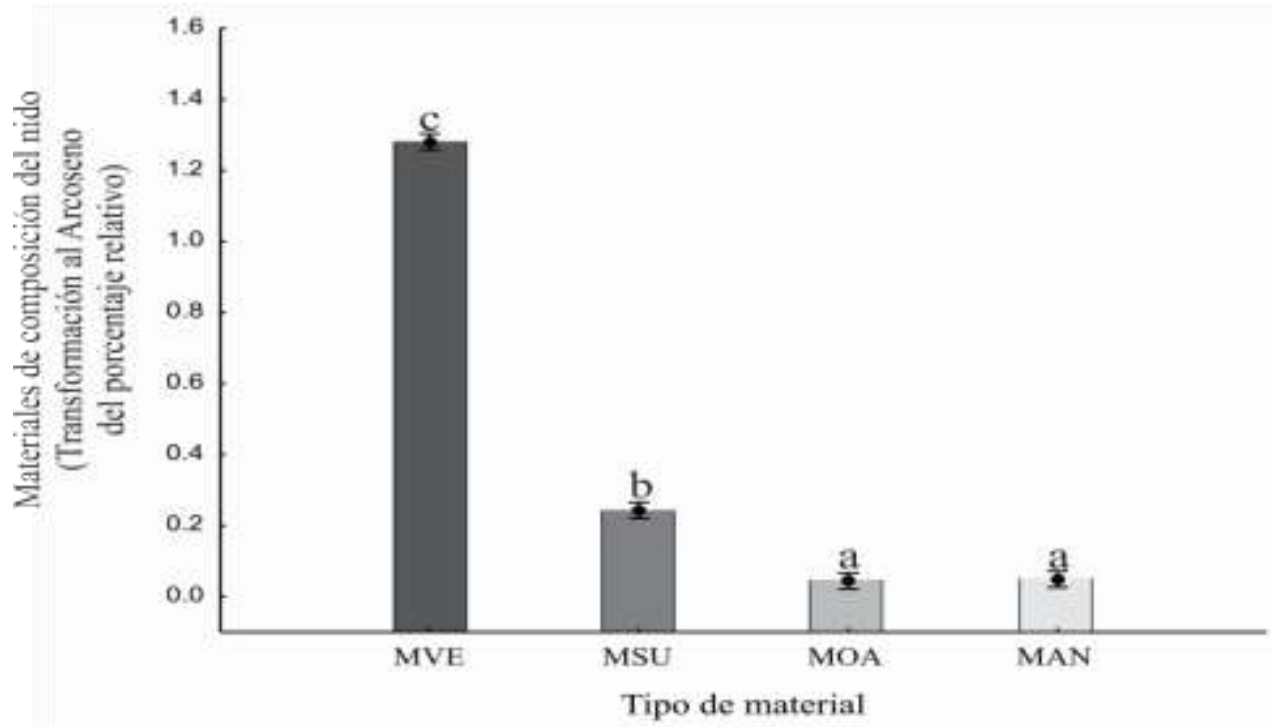

Figura 1. Diferencias significativas detectadas en la composición de nidos de aves silvestres por tipo de material provenientes de parques urbanos y periurbanos de las ciudades de Xalapa y Coatepec, Veracruz, México. Letras diferentes indican diferencias significativas $\mathrm{P}<0.05$. MVE: Material vegetal; MSU: material de sustrato; MOA: material de origen animal; MAN: material de origen antrópico.

Tabla 2. Matriz de correlación descriptivaque muestra la variable, tipo de material con los componentes principales y sus valores propios obtenidos por ACP y el porcentaje de variabilidad mostrado en cada componente. MVE: Material vegetal; MSU: material de sustrato; MOA: material de origen animal; MAN: material de origen antrópico.

\begin{tabular}{lll}
\hline Componentes del nido & PC1 & PC2 \\
\hline Valores propios & 2.2203 & 1.5077 \\
\hline MVE & 0.40 & 0.62 \\
MSU & -0.49 & 0.48 \\
MOA & 0.56 & 0.39 \\
MAN & -0.51 & 0.46 \\
\hline Variabilidad explicada \% & 55.50 & 37.70
\end{tabular}

Se sabe que la mayoría de aves construyen sus nidos con componentes vegetales, como corteza, ramas, pastos y musgos (Hansell, 2000). Considerando esto, Clark y Mason (1988) mencionaron que el uso regular de material vegetal por aves en sus nidos está relacionado con el éxito reproductivo y el potencial de reclutamiento. Esto se basa en que al contar con volátiles o compuestos secundarios que actúan como insecticidas favorecenla sobrevivencia de los polluelos y la salud del nido. Bajo este hecho y con base en el comportamiento observado en diversas especies de aves. Clark (1991) propuso la hipótesis de protección del nido, que señala que algunos de los materiales vegetales frescos utilizados por las aves en sus nidos contienen compuestos secundarios que repelen ectoparásitos y microparásitos, como bacterias, que enmascaran las señales químicas que éstos utilizan para encontrar el hospedero y permiten la sobrevivencia de los polluelos estando así 
involucrados con la salud del nido (Pires et al., 2012; Ruiz-Castellano, 2016). Esto ha sido comprobado en diversos estudios. Por ejemplo, miembros de la familia Paridae, utilizan plantas aromáticas como Achillea ligustica, Helichry sumitalicum, Lavandula stoechas y Cistus creticus en sus nidos (Lafuma et al., 2001) y rapaces, como Buteo lineatus material vegetal de cuatro especies de coníferas en sus nidos, las que son ricas en componentes secundarios (Dykstra et al., 2009). En este estudio, se identificó que uno de los materiales vegetales presente en los nidos de dos de los parques periurbanos estudiados (El Haya y el Francisco Javier Clavijero) fueron las acículas de pinos, las que al contener resinas, permiten pensar en su papel como elementos antiparasitarios y antibacterianos, particularmente por su contenido de alcaloides (Martin et al., 2002). Aves del género Sitta, por ejemplo, cubren las cavidades del nido con resina de pino (Bent, 1964), al contener monoterpenos con propiedades antisépticas (Rosenthal y Janzen, 1979).

Un estudio con el herrerillo común Cyanistes caeruleus documentó que el uso de plantas aromáticas disminuye el riesgo de picadura del mosquito Culex pipiens, principal vector de la malaria aviar (Scott y Edman, 1991), así como de otros mosquitos de los géneros Aedes, Ochlerotatus y Culiseta (Rioux et al., 1974). Con la misma especie (Cyanistes caeruleus), un estudio realizado en el parque forestal de Monsanto en Lisboa-Portugal en bosques de Quercu ssp. y Pinus pinea, se documentó el uso de tres especies aromáticas con fines de protección en los nidos como Dittrichia viscosa, Lavandula dentata y Calamintha baetica (Pires et al., 2012). Por otro lado, el estornino pinto (Sturnus vulgaris) usó siete plantas aromáticas, como Brachypodium silvaticum, Aegopodium podagraria, Heracleums phondylium, Sambucus niger, Anthriscus sylvestris, Achillea millefolia y Salix alba en los nidos, y se encontró que estas plantas favorecían el sistema inmunológico de los polluelos y su resistencia ante la presencia de ectoparásitos (Gwinner et al., 2000).

De acuerdo con Hansell (2000), la selección de material vegetal por aves está también involucrada con aspectos de la firmeza y estabilidad del nido, al ser tallos, ramas y pastos, los que dan forma al cuenco, así como con aspectos de termorregulación. Nidos con bajo material térmico pueden implicar un mayor gasto energético y tiempo de los padres para tratar de incubar sus huevos (Canestrari et al., 2007); por lo que, las aves usan más material de tipo celuloso, como fibras vegetales que permiten una rápida pérdida de humedad al sol o por acción del viento (Attenborough, 1998). La fibra vegetal, estuvo altamente representada en los nidos de este estudio lo cual sugiere que se usa como elemento principal para el revestimiento exterior e interior del nido por sus propiedades térmicas. Así también, la arcilla fue un elemento importante en los nidos. Se ha documentado que este material tiene buenas propiedades estructurales al ser fácilmente moldeable, brindando así firmeza y solidez (Kilgore y Knudsen, 1977), el que pudiera estar siendo usado como material secundario de soporte. La presencia de mayor material vegetal y de sustrato, muestra que estos parques urbanos y periurbanos de Xalapa y Coatepec cuentan con los materiales necesarios para satisfacer las demandas estructurales y de revestimiento de aves silvestres en la construcción de sus nidos. Estudios que evalúen cómo las aves usan diferentes materiales para la composición de sus nidos pueden ser útiles para identificar el impacto inmediato de fenómenos como la 
urbanización (Chace y Walsh, 2006; Hanmer et al., 2017).

\section{CONCLUSIÓN}

Aves silvestres consideran mayoritariamente el uso de material vegetal y de sustrato para la construcción de sus nidos independientemente del tipo de parque urbano o periurbano, lo cual sugiere que éstos son de importancia para aspectos de soporte y revestimiento. Sin embargo, la presencia de material antrópico en mayor o menor proporción puede ser un indicador de riesgo para las aves y sus polluelos $\mathrm{y}$ afectar negativamente su reclutamiento en áreas urbanas.

\section{LITERATURA CITADA}

Attenborough, D. 1998. The life of birds. London: BBC Books. RSK Barnes and RN Hughes.Trends in Ecology and Evolution 13: 70-74.

Bent, A. C. 1964. Life histories of North American nuthatches, wrens, thrashers and their allies. Reprinted from Smithsonian Institution Unite States National Museum. Dover Publications, Inc., New York, pp: 948.

Bondia-López, B. 2014. Composición del nido del gorrión molinero (Passer montanus) y su adaptación a entornos urbanos. Tesis de grado. Universidad Complutense de Madrid, España, pp: 28. Canestrari, D., Marcos, J. M. y Baglione, V. 2007. Costs of chick provisioning in cooperatively breeding crows; an experimental study. Animal Behaviour 73: 349-357.

https://doi.org/10.1016/j.anbehav.2006. 04.013
Chace, J. F. y Walsh, J. J. 2006. Urban effects on native avifauna: a review. Landscape Urban Plan 74: 46-69.

https://doi.org/10.1016/j.landurbplan.200 4.08.007

Clark, L. 1991. The nest protection hypothesis: the adaptive use of plant secondary compounds by European starlings. Bird-parasite interactions: ecology, evolution and behaviour. Oxford University Press, Oxford, pp: 221.

Clark, L. y Mason, J. R. 1985. Use of nest material as insecticidal and antipathogenic agents by the European starling. Oecologia 67(2): 169-176. https://doi.org/10.1007/BF00384280

Collias, N. y Collias, E. 1984. Nest building behavior in birds. Princeton University Press, New Jersey, pp: 358. https://doi.org/10.1515/9781400853625

Dykstra, C. R., Hays, J. L. y Simón, M. M. 2009. Selection of fresh vegetation for nest lining by red-shouldered hawks. The Wilson Journal of Ornithology 121: 207-210. https://doi.org/10.1676/08-035.1

Estades, C. F. 1995. Aves y vegetación urbana: el caso de las plazas. Boletín Chileno de Ornitología 2: 7-13. 
Gwinner, H., Oltrogge, M., Trost, L. y Nienaber, U. 2000. Green plants in starling nests: effects on nestlings. Animal Behaviour 59(2): 301-309. https://doi.org/10.1006/anbe.1999.1306

Hanmer, H. J., Thomas, R. L., Beswick, G. J. F., Collins, B. P. y Fellowes, M. D. E. 2017. Use of anthropogenic material affects bird nest arthropod community structure: influence of urbanisation, and consequences for ectoparasites and fledging success. Journal of Ornithology 158(4): $\quad$ 1045-1059. https://doi.org/10.1007/s10336-017-1462 $-7$

Hansell, M. 2000. Bird Nest and Construction Behaviour. Cambridge University Press, Cambridge, $\quad$ pp: 280. https://doi.org/10.1017/CBO9781139106 788

Kilgore, Jr. D. L. y Knudsen, K. L. 1977. Analysis of materials in Cliff and Barn Swallow nests: relationship between mud selection and nest architecture. The Wilson Bulletin 89(4): 562-571.

Lafuma, L., Lambrechts, M. M. y Raymond, M. 2001. Aromatic plants in bird nests as a protection against blood-sucking flying insects? Behavioural Processes, 56(2): 113-120.

https://doi.org/10.1016/S0376-6357(01)0 0191-7

Martin, D., Tholl, D., Gershenzon, J. y Bohlmann, J. 2002. Methyl jasmonate induces traumatic resin ducts, terpenoid resin biosynthesis, and terpenoid accumulation in developing xylem of Norway spruce stems. Plant physiology 129(3):

1003-1018. https://doi.org/10.1104/pp.011001
Moreno, J, S., Vásquez, M. y Armesto, J. 2005. Breeding biology of the thorn-tailed rayadito (Furnariidae) in south temperate rainforests of Chile. The Condor 107 : 69-77. https://doi.org/10.1093/condor/107.1.69

Niemelä, J. 2000. Biodiversity monitoring for decision-making. Annales Zoologici Fennici 37: 307-317.

Parker, G. H. y Blomme C. G. 2007. Fish-line entanglement of nesting mourning dove, Zenaida macroura. The Canadian FieldNaturalist 121(4): 436-437. https://doi.org/10.22621/cfn.v121i4.522

Pires, B. A., Belo, A. F. y Rabaça, J. E. 2012. Aromatic plants in Eurasian Blue Tit nests: the 'nest protection hypothesis' revisited. The Wilson Journal of Ornithology 124: 162-165. https://doi.org/10.1676/11-102.1

Quilodrán, C., Vásquez, M. y Estades, V. 2012. Nesting of the Thorn Tailed Rayadito (Aphrasturas pinicauda) in a Pine Plantatio in Southcentral Chile. The Wilson Journal of Ornithology 124: 737-742.

https://doi.org/10.1676/1559-4491-124.4. 737

Rioux, J. A., Cousserans, J., Croset, H. y Gabinaud, A. 1974. Présence du caractere autogenedans les populationsd',Aedes (O.) detritus (Haliday, 1833) du 'Midi'méditerranéen et de la Corse. Annales de Parasitologie Humaine et Comparée 49: 129-130.https://doi.org/10.1051/parasite/ 1974491129

Rosenthal, G. A. y Janzen, D. H. 1979. Herbivores: Their interaction with secondary plant metabolites. Academic Press, New York, pp: 493. 
Ruiz-Castellano, C. 2016. Plumas, plantas y bacterias en nidos de estornino negro (Sturnus unicolor) y sus efectos en la reproducción. Tesis Doctorado. Universidad de Granada. Departamento de Zoología. Granada, España, pp: 299. https://doi.org/10.7818/ECOS.2017.26-2.07

Scott, T. W. y Edman, J. D. 1991. Effects of avian host age and arbovirus infection on mosquito attraction and bloodfeeding success. In: Loye, J. E. y Zuk, M. (Eds.), Bird-Parasite Interactions. Ecology, Evolution, and Behaviour. Oxford University Press, Oxford, 179204.
Suárez-Rodríguez, M., Rull, I. L. y MacíasGarcía, C. 2013. Incorporation of cigarette butts into nests reduces nest ectoparasite load in urban birds: new ingredients for an old recipe?. Biology Letters 9(1): 20120931. https://doi.org/10.1098/rsbl.2012.093

Townsehd, A. K. y Barker, C. M. 2014.Plastic and the nest entanglement of urban and agricultural crows. PLoS ONE 9(1): e88006.

https://doi.org/10.1371/journal.pone.0 088006

Zar, J. H. 1999. Biostatistical analysis, 4.Ed. Prentice Hall, New Jersey, pp: 960.

Copyright (c) 2018 Rafael Flores Peredo, Xóchitl Zárate Tirado, Beatriz del Socorro Bolivar Cimé, Isaac Mella Méndez, Maria del Coral Rosas Ronzón, Paola Ivette Dominguez Espinosa

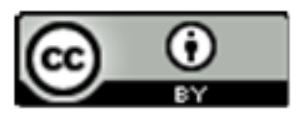

Este tex to está protegido por una licencia CreativeCommons $\underline{4.0}$

Usted es libre para Compartir — copiar y redistribuir el material en cualquier medio o form ato- y Adaptar el documento —remezclar, transformar y crear a partir del material- para cualquier propósito, incluso para fi nes comerciales, siempre que cumpla la condición de:

Atribución: Usted debe dar crédito a la obra original de manera adecuada, proporcionar un enlace a la licencia, e in dicar si se han realizado cam bios. Puede hacerlo en cualquier form a razonable, pero no de form a tal que sugiera que tiene el apoyo del licenciante o lo recibe por el uso que hace de la obra. 